\section{Creating a safety culture in academic laboratories}

\author{
Gary L. Messing
}

W hen we began the process of raising our laboratory safety standards in the Department of Materials Science and Engineering (MatSE) at The Pennsylvania State University (Penn State) in 2008, we had no idea the implementation of an effective safety program would be so challenging. But changing a culture takes more than lip service; it requires an unequivocal commitment of time, strong leadership, resources, and ideas from staff, faculty, and students. Now our efforts of enhanced safety awareness are spreading across the Penn State campus, and as a result of our experience, we have been able to help other laboratories and organizations accelerate the change from a safety-when-convenient perspective, that is, the old way, to a safety-first mentality - the only way forward.

The process for change began when, despite our department's already heavily invested efforts in laboratory safety, we realized the inadequacy of our approach. The highly publicized tragedy in a chemistry laboratory at the University of California-Los Angeles in 2008, in which a 23-year-old research assistant lost her life due to inadequate training and safety practices, ${ }^{1}$ only served to bring into sharper focus the need for a total commitment to making safety as high a priority for our department as research success has always been.

Among our first steps was to reach out to our external advisory board (EAB), a group of leaders from industry, academia, and government laboratories. Input from industry and government laboratory leaders was crucial, as they embrace safety in a way that the best of academia has not. The EAB members brought with them the rigorous safety practices and worker training programs that are typical in industry laboratories,

Gary L. Messing, The Pennsylvania State University along with an attitude of safety first and constant evaluation and improvement of safety practices. They advised us on ways to improve our physical facilities, train laboratory personnel, maintain safety documentation, and instill an approach to research that is steeped in safety.

In addition, Owens Corning and Dow Chemical provided their experts to evaluate our safety practices and to give us advice on how to effect real change. James Loftus from Owens Corning and chair of the EAB is a vocal participant on our board and is enthusiastic about bringing the company's experiences with safety to Penn State. He gave a seminar to our graduate students about the importance of safety and invited Donald Hurd, the Owens Corning safety officer, to review our laboratories and processes. Our EAB members also provide inspections during the semi-annual board meetings to provide us with direct feedback about weaknesses and strengths in our safety procedures. Dow Chemical's involvement was prompted by a recently initiated research program at Penn State. They wanted to ensure that their sponsored research was being conducted in a safe manner commensurate with the level of safety practiced in their own laboratory facilities as a part of their well-publicized outreach program in university safety. ${ }^{2,3}$

Our industry advisors made it clear that in their laboratories, safety is a nonnegotiable requirement and is their first priority when entering a laboratory. This fact is made clear when our students visit potential employers on our annual industry field trips. The most recent tour, led by MatSE assistant professor Joshua Robinson, is a case in point. The trip was an eye-opener, for both Robinson and the students. They toured five manufacturing facilities producing specialty metals, polymers, ceramics, chemical supplies, and semi- conductors. The first order of business at each plant was a safety orientation that included proper use of safety equipment for the tour. At the polymers company, Robinson said, the vice president for research pointed out that the student summer interns they hired had good research skills but failed when it came to adequately knowing safety procedures. He called it "a strike against them" in terms of hiring because companies are intolerant of unsafe practices. Industry wants and expects safety to be taught at universities, beginning at the undergraduate level, Robinson was told.

Robinson is now implementing the practices developed in MatSE at the Materials Research Institute's laboratories in the Millennium Science Complex (MSC), an interdisciplinary research building that houses faculty and graduate students from the MatSE, electrical engineering, mechanical engineering, chemistry, physics, and bioengineering departments. MSC has a diverse mix of technologies and practices, made even more challenging to manage safety-wise by the busy user facilities open to onand off-campus researchers.

"Our idea is to motivate students to think about safety," Robinson said. "It's not about punishment, but more about 'look how important this is and how you can make a difference."”

To that end, the safety committee has created eye-catching safety signage for all of the laboratories featuring emergency contact numbers, quick reference guides on what to do in the event of an injury or chemical spill, and reminders of good safety practices. Another initiative promoted in MatSE and brought to the MSC is Stall Wall News in restrooms - a monthly update of short, informative articles and news items about lab safety. The goal is to surround students and faculty with safety information so that thinking about safety becomes second nature.

Over the last five years, the MatSE Safety Awareness Organization (MSAO), which was created as one of the first actions in the safety campaign, has been responsible for creating programs to raise safety awareness, such as a drive to pro- 
vide safety glasses to all researchers, monthly laboratory inspections, safety posters for the laboratories, Safety Moment presentations to begin all department seminars, a monthly safety newsletter, a safety web page on the department website with links to important safety sites, and a safety video. Examples of safety initiatives that have been implemented include:

- Placards developed by the MSAO as reminders about personal protective equipment rules. Each laboratory door has a sign on the outside that reads "safety glasses required beyond this point" and another on the inside reminding researchers that gloves must be removed before exiting the lab.

- "Safety First" signs (see figure) posted in each laboratory with a quick guide on handling emergency situations like chemical spills, fires, and injuries; safety training requirements; and a list of phone numbers for Penn State's Environmental Health and Safety (EHS), campus police, department and college safety officers, and medical facilities.

- Clothing requirements for anyone working in a MatSE laboratory: long pants and close-toed shoes, in addition to the personal protective equipment requirements of safety glasses and lab coats, at a minimum, plus gloves that are rated for the chemicals being handled, respiratory protection (dust masks up through respirators), and/or goggles or face shields, as necessary.

The MSAO also initiated "Safety Week" at the beginning of the fall 2012 semester, with experts from Penn State's EHS office and industry partners who are brought in to make presentations on critical safety issues and to give demonstrations about chemical waste handling, fire extinguisher training, and chemical inventory procedures. All of our faculty, staff, and students, whether they regularly work in a laboratory or do computational work in an office, are required to take this training annually.

A key element in the success of creating a safety culture is the constant en- couragement and support of the leadership team, which provides staff support, faculty time, and financial backing to pursue safety as a departmental priority. As we have been taught by industry, developing a true safety culture transcends the simple implementation of policies and procedures already in place - it is a change in mind-set and action and a commitment to make safety an integral part of doing business for the long term. That is, a change in culture.

The proper staffing of a safety team is critical. Michael Hickner, a new assistant professor from Sandia National Laboratories when we started our safety initiative, was appointed as the faculty member in charge of the MatSE Safety Awareness Organization. The US national laboratories are known to have a strong safety culture and safety training programs, so Hickner was able to bring that experience to Penn State. In addition, the technical staff member on the team, Scott Henninger, has been crucial in providing daily communications about best practices such as safety training, sample labeling, housekeeping, proper use of personal protective equipment, and updating documentation to both faculty and students. Currently, three graduate students and two undergraduate team members are our idea generators, peer support, and the barometers for student responses in the laboratory.

Recently, Penn State, along with the University of Minnesota and the University of California-Santa Barbara, partnered with Dow Chemical Company to transition Dow's industrial safety culture to other university laboratories. The pilot program began last summer, with biweekly meetings between representatives from Dow and an Interdepartmental Safety Team from MatSE, Chemistry, and Chemical Engineering at Penn State. Dow's program of wide engagement with universities on safety is unique, but we hope other companies mirror their efforts with their own affiliated schools. Engaging with industry and with other universities also provides a measure of accountability in our department to make sure we

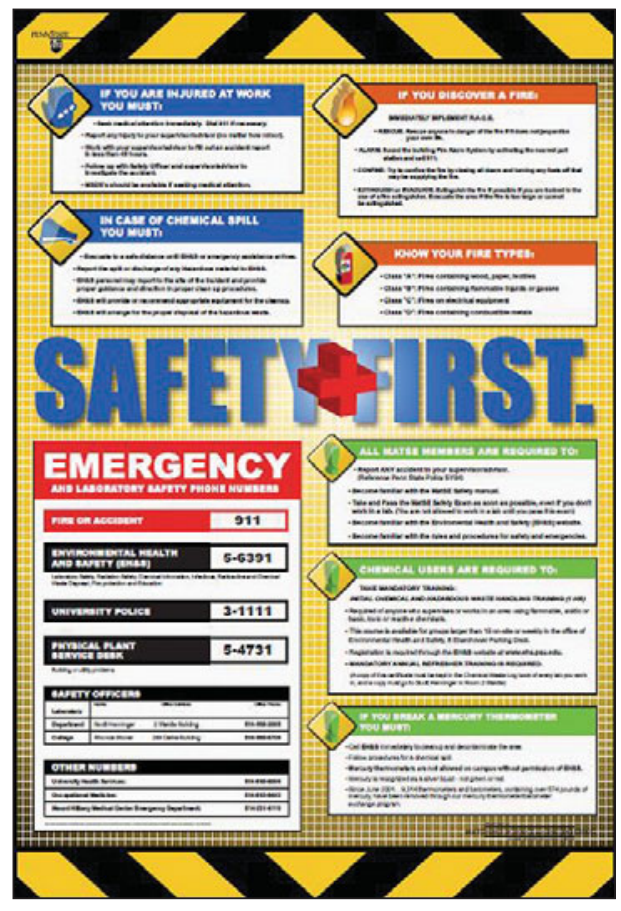

The Department of Materials Science and Engineering at The Pennsylvania State University places "Safety First" signs in each laboratory as part of its mission to make "safety" second nature.

continue to provide as safe a working environment as possible.

Now that we have developed what we feel is one of the strongest safety programs in academia, we believe our experience could help others accelerate the building of a strong safety culture at their universities. We are planning a safety tutorial at the 2014 Materials Research Society Spring Meeting, titled, "Safety First: Enhancing Safety in Academic Research Laboratories." We welcome inquiries through our departmental website at www.MatSE.psu. edu/safety.

\section{Additional Information}

Safety in the lab video

http://bit.ly/13h8Tu2

\section{End Notes}

1. http://cen.acs.org/articles/90/i5/SystemicFailures-Cited-Lab-Death.html (accessed 4/8/13). 2. http://cen.acs.org/articles/90/i44/Dow-ChemicalTeams-Universities-Laboratory.html (accessed 3/21/13).

3. http://cenblog.org/the-safety-zone/2012/05/dowand-minnesota-team-up-on-safety/ (accessed $3 / 21 / 13)$. 